\title{
基于浮油膜双光束干涉模型的油膜厚度遥感 反演理论
}

陆应诚 ${ }^{(1)}$, 田庆久 ${ }^{(1)}$, 李想 ${ }^{2}$

(1) 南京大学国际地球系统科学研究所, 南京 210093 ;

(2) 南京大学电子科学与工程学院, 南京 210093

*E-mail: lycheng2003@ sina.com

收稿日期: 2010-06-03; 接受日期: 2010-11-02

国家自然科学基金(批准号: 40971186, 41001196)、中国科学院数字地球重点实验室开放基金项目(编号: 2010LDE007)资助

摘要 海面浮油膜是海洋石油污染监测与海洋油气资源勘探的共同关注对象, 其厚度的遥感 定量反演是研究难点之一。随着油膜厚度的变化, 入射光在浮油膜中的辐射传输有所不同, 是 海面浮油膜厚度光学遥感反演的理论依据. 本研究将均匀分布的浮油膜视为一个平行平板, 分 析其可见光范围内的辐射传输过程，探讨油膜反射光的双光束干涉，构建以油膜厚度及其光谱 反射率为变量的油膜厚度遥感定量反演模型, 并结合菲涅耳公式进行模型参数的物理意义分析, 揭示油膜厚度遥感定量反演的关键在于油膜的消光特性. 利用油膜光谱响应实验数据, 开展理 论模型的分析与验证, 结果表明: 油膜厚度遥感定量反演的理论模型不仅具有明确的光学物理 意义, 同时也具有较好的模拟精度; 浮油膜光谱反射率随厚度变化响应的主要因素是油膜对入 射光的分子散射作用，该散射作用与入射光波长具有显著的关系，因此油膜光学遥感散射特性 研究将有助于油膜种类识别与厚度的遥感定量反演; 模型参数的理论意义与数据分析表明, 建 立典型油种油膜的模型参数查找表, 将有效提高海面浮油膜厚度遥感定量反演的效率.

关键词

双光束干涉 油膜厚度 遥感 反射率
海面浮油膜主要有人为与自然两种来源, 人为 来源是人类在石油开采、加工、运输等过程中的溢油 事故产生的海面浮油膜; 天然来源主要是海底具有 工业开采价值的海洋油气藏的烃渗漏逐渐形成 ${ }^{[1 \sim 3]}$, 据估算海洋烃物质中大约 53\%的来自人类活动, $47 \%$ 来源于自然原因 ${ }^{[4]}$. 海面浮油膜一直为海洋石油污染 监测与海洋油气资源勘探共同关注, 多/高光谱 ${ }^{[5 \sim 14]}$ 、 热红外 ${ }^{[15,16]}$ 、微波雷达 ${ }^{[17 ~ 19]}$ 、激光苂光 ${ }^{[20 ~ 23]}$ 等诸多 遥感技术都对此有了较深的研究. 早期研究因对海 面油膜存在状态、类型等的认知差异, 研究的结果也
多有不同 ${ }^{[2426]}$, 针对厚油膜状态时的研究较多, 厚 油膜对入射光较强吸收、低穿透性、低反射性等特点, 因而反射光谱形态单一, 且缺乏明显的光谱特征. 随 着对海面油膜存在状态认知的深入, 高光谱遥感的 技术优势得到体现. Palmer 等 ${ }^{[5]}$ 利用小型机载成像光 谱仪(CASI)数据分析了 1993 年 1 月 5 日发生于英国 设得兰(Shetlands)群岛的溢油事件, 从精细光谱分辨 率的角度探讨了不同类型海面石油污染的光谱特征, 研究表明 440 900 $\mathrm{nm}$ 是可以用来进行溢油油膜信 息提取的有效谱段. 赵冬至和从丕福 ${ }^{[7]}$ 通过实验，总

英文引用格式: Lu Y C, Tian Q J, Li X. The remote sensing inversion theory of offshore oil slick thickness based on a two-beam interference model. Sci China Earth Sci, 2011, 54: 678-685, doi: 10.1007/s11430-010-4154-1 
结了柴油、润滑油和原油 3 种油膜的可见光近红外波 段地物光谱特征, 为识别油膜厚度、区分油膜与海水 和鉴别油种提供最初的研究参考. 张永宁等 ${ }^{[8,9]}$ 分析 了海洋溢油波谱特征的测试结果, 认为溢油波谱特 征除受外部环境因素影响外, 与溢油种类和油膜厚 度有密切的关系, 实验表明 500 580 nm 是不同油膜 最高反射率的所在位置. Foudan ${ }^{[6]}$ 利用具有 224 个连 续波段, 光谱覆盖范围为 380 2500 $\mathrm{nm}$ 的机载可见 光/近红外成像光谱仪(AVIRIS)研究了位于美国圣巴 巴拉(Santa Barbara)海岸带的溢油, 表明油量的增加 导致光的吸收加大, 从而降低了可见光波段的反射 率, 指出反射红外 600 900 $\mathrm{nm}$ 具有最大的油膜遥感 探测可能性. 近年来, 陆应诚等 ${ }^{[11,12]}$ 通过海面薄油膜 光谱实验将海面油膜划分为甚薄油膜与海面薄油膜 等, 研究指出海面甚薄油膜具有增反效应, 因此光谱 反射率会高于本底水体; 油膜厚度连续变化的光谱 响应实验中, 油膜的光谱反射率与厚度呈很强的幂 函数负相关关系, 认为绿光、红光波段可作为海面油 膜多/高光谱遥感探测与评估的最佳选择波段, 同时 研究也进一步指出碳氢化合物反射光谱因其电子跃 迁与物分子基频振动的特点, 海面碳氢化合物缺乏 明显的反射光谱特征. 此后, 付玉慧等 ${ }^{[13]}$ 也分析了不 同油种海面油膜光谱特性, 研究结论进一步充实了 海面油膜光谱特性的分析. 2009年, Magnus 等 ${ }^{[14]}$ 开展 了海面油膜光谱实验, 深入分析海面油膜厚度变化 的反射光谱响应, 同时开展了油膜厚度的估算研究, 取得较好的效果. 该研究表明海面薄油膜的反射光 谱响应差异能为海面薄油膜的识别与定量反演提供 技术支持. 已有的研究表现出以下几个特点与趋势: 随着对海面油膜来源、构成、类型、状态的认知深入, 高光谱遥感探测海面油膜的技术优势得以体现; 海 面油膜厚度变化导致的反射光谱响应差异与变化成 为研究的重点; 绿光-红光波段作为海面油膜多/高光 谱遥感探测与评估的最佳选择波段成为共识; 不同 背景环境、油种、实验条件等情况下的海面油膜光谱 响应个性差异研究, 逐渐深入到对其光辐射传输过 程的共性研究.

本文将海面浮油膜视为一层平行平板, 探讨入 射光在其中辐射与传输过程, 构建油膜反射光的双 光束干涉模型, 研究油膜厚度遥感反演理论; 结合油 膜光谱响应实验获取的油膜厚度与反射光谱数据, 开展模型的数据分析与验证, 进一步指出油膜厚度
遥感反演理论中油膜对入射光的分子散射特性与意 义, 为海面浮油膜厚度的遥感研究奠定理论基础与 方法参考。

\section{1 油膜反射光的双光束干涉}

平静海面上均匀分布的海面浮油膜, 有空气、油 膜和海水三种介质层, 三种介质的折射率明显不同, 入射光会在油膜层内发生折射与反射, 因此水表面 的浮油膜层可视为均匀的平行平板 (如图 1 所示), 上 面第一层是空气层, 其折射率为 $n_{1}$; 中间一层是浮油 膜层, 油膜的折射率为 $n_{2}$, 油膜厚度为 $d$; 最下面一 层为海水层, 其折射率为 $n_{3}$. 入射光从空气层入射到 油膜层, 入射光的原子发光持续时间很短, 不同时间 的光之间的相关性较低, 即相干长度极短, 该入射光 的平行平板干涉, 可以简化为双光束干涉 ${ }^{[27,28]}$.

设入射光电矢量强度为 $\vec{E}_{0}$, 波数为 $k=2 \pi / \lambda$; 入 射光从空气层射入油膜层时, 油膜上表面透射率为 $T_{12}$ 、反射率为 $R_{12}$; 油膜层内的透射光射入油膜与海 水界面时, 即射入油膜层下表面时, 反射率为 $R_{23}$; 油膜层内透射光经过油膜下表面反射后, 从油膜层 的上表面透射入空气时, 其透射率为 $T_{21}$. 如图 1 所示, 则入射光在油膜上表面的第一次反射的电矢量强度 $\vec{E}_{1}=R_{12} \vec{E}_{0}$; 第一次透射入油膜的光电矢量强度为 $T_{12} \vec{E}_{0}$, 油膜层中该透射光经油膜下表面反射后光的 电矢量强度为 $T_{12} R_{23} \vec{E}_{0}$; 油膜层中透射光经油膜下表 面的反射, 又经过油膜上表面透射至空气层, 其电矢 量强度为 $\vec{E}_{2}$, 考虑到 $\vec{E}_{1}$ 和 $\vec{E}_{2}$ 在传播路径上有长度为 $\Delta$ 的差别, 两束光之间则存在相位延迟 $K \Delta$, 若光束在 油膜层传播过程中的消光系数为 $\alpha$, 从油膜下表面

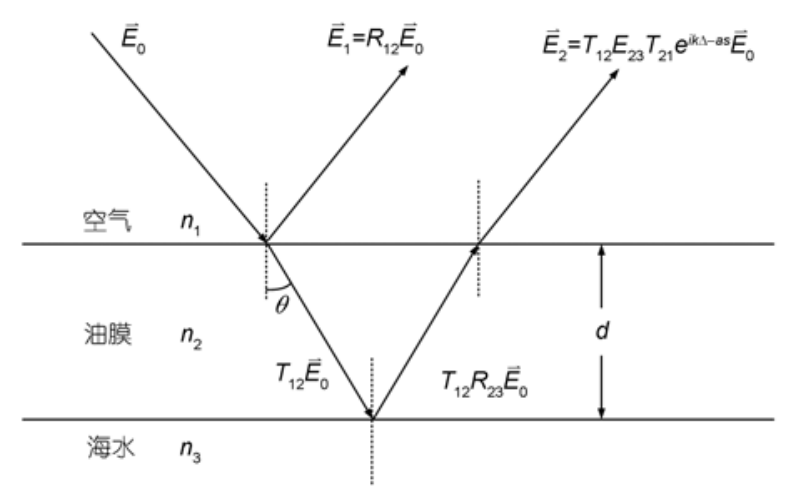

图 1 油膜反射光的双光束干涉 
反射回空气的光束在油膜中传播路径长度为 $S$, 则透 射光电矢量强度为 $\vec{E}_{2}=T_{12} R_{23} T_{21} \mathrm{e}^{i k \Delta-a s} \vec{E}_{0}$. 平行平 板干涉也就是反射光 $\vec{E}_{1}$ 与油膜出射光 $\vec{E}_{2}$, 两者的电 矢量叠加后产生双光束干涉. 在探测器的焦平面上, $\vec{E}_{1}$ 与 $\vec{E}_{2}$ 叠加产生干涉, 进入遥感器系统的电矢量 $\vec{E}=\vec{E}_{1}+\vec{E}_{2}$, 则油膜反射率 $R$ 可以计算得到, 即

$$
R=\frac{|\vec{E}|^{2}}{\left|\vec{E}_{0}\right|^{2}}=\frac{\left|\vec{E}_{1}+\vec{E}_{2}\right|^{2}}{\left|\vec{E}_{0}\right|^{2}}=\left|R_{12}+T_{12} R_{23} T_{21} \mathrm{e}^{i k \Delta-a s}\right|^{2},
$$

其中

$$
\left\{\begin{array}{l}
\Delta=2 n_{2} d \cos \theta, \\
s=\frac{2 d}{\cos \theta} .
\end{array}\right.
$$

公式(1)可以展开为

$$
R=\left(R_{12}\right)^{2}+2 R_{12} T_{12} R_{23} T_{21} \cos (k \Delta) \mathrm{e}^{-a s}+\left(T_{12} R_{23} T_{21}\right)^{2} \mathrm{e}^{-2 a s} .
$$

公式(3)描述了油膜反射率与油膜厚度、入射光折射 角、各界面反射率和透过率之间的理论关系.

\section{2 模型与参数分析}

将公式(2)和(3)表述为油膜厚度 $d$ 与油膜反射率 $R$ 的遥感定量模型, 分析每一个参数的理论意义.

设:

$$
\left\{\begin{array}{l}
A_{0}=\left(R_{12}\right)^{2}, \\
A_{1}=2 R_{12} T_{12} R_{23} T_{21}, \\
A_{2}=\frac{\alpha s}{d}, \\
A_{3}=\frac{k \Delta}{d}, \\
A_{4}=\left(T_{12} R_{23} T_{21}\right)^{2},
\end{array}\right.
$$

则公式(3)可写为

$$
R=A_{0}+A_{1} \mathrm{e}^{-A_{2} d} \cos \left(A_{3} d\right)+A_{4} \mathrm{e}^{-2 A_{2} d} .
$$

从公式(4)和(5)中可知, $A_{0}, A_{1}, A_{4}$ 与各界面反射 系数和透射系数相关, $A_{2}$ 和 $A_{3}$ 与各界面的反射系数 和透射系数无关. 结合公式(2)对 $A_{2}$ 和 $A_{3}$ 展开得

$$
\begin{gathered}
A_{2}=\frac{a s}{d}=\frac{2 a}{\cos \theta}, \\
A_{3}=\frac{k \Delta}{d}=2 k n_{2} \cos \theta .
\end{gathered}
$$

从公式(6)和(7)可见, 参量 $A_{2}$ 与油膜的消光系数 有密切关系, 参量 $A_{3}$ 与油膜折射率有关系, 对固定 油种的油膜来说, 该折射率是固定值. 如假设入射角 不变, 则折射角也是固定的, 因此参数 $A_{3}$ 可以视为 一个常数. 所有参量中, $A_{0}, A_{1}, A_{3}$, 和 $A_{4}$ 只与入射光 入射角、偏振特性以及介质的物理特性有关，这些参 量与探测方式, 形成油膜的油种等有关, 因此这 4 个 参量可以认为是模型常量; 参量 $A_{2}=\alpha s / d$, 该参量中 有油膜对入射光的消光系数 $\alpha$, 油膜厚度的变化使其 对入射光的消光也发生了变化.

在浮油膜双光束干涉模型中, 油膜界面上的反 射率和透射率系数可以通过菲涅耳(Fresnel)公式计算. 若入射角为 $\theta_{1}$, 反射角也为 $\theta_{1}$, 透射角为 $\theta_{2}$, 入射光 部分的介质折射率为 $n_{1}$, 磁导率为 $\mu_{1}$, 透射光部分的 介质折射率为 $n_{2}$, 磁导率为 $\mu_{2}$. 入射平面为入射光束 方向和界面法线形成的平面, 可以将入射光电矢量 按垂直于入射平面和平行于入射平面分解成两个正 交的分量, 如图 2 所示, 入射光的磁感应强度为 $\vec{B}$, 电场强度为 $\vec{E}$, 反射光的磁感应强度为 $\vec{B}^{\prime \prime}$, 电场强 度为 $\vec{E}^{\prime \prime}$, 透射光的磁感应强度为 $\vec{B}^{\prime}$, 电场强度为 $\vec{E}^{\prime}$ ，下标 $p$ 表示振动方向平行于入射面分量、下标 $s$ 表示振动方向垂直于入射面分量 ${ }^{[27]}$.

入射光电矢量平行于入射平面的 $p$ 分量, 反射系 数 $r_{p}$ 和透射系数 $t_{p}$ 为

$$
\begin{gathered}
r_{p}=\frac{\vec{E}_{p}^{\prime \prime}}{\vec{E}_{p}}=\frac{\frac{\mu_{1}}{\mu_{2}} n_{2}^{2} \cos \theta_{1}-n_{1} \sqrt{n_{2}^{2}-n_{1}^{2} \sin ^{2} \theta_{1}}}{\frac{\mu_{1}}{\mu_{2}} n_{2}^{2} \cos \theta_{1}+n_{1} \sqrt{n_{2}^{2}-n_{1}^{2} \sin ^{2} \theta_{1}}}, \\
t_{p}=\frac{\vec{E}_{p}^{\prime}}{\vec{E}_{p}}=\frac{2 n_{1} n_{2} \cos \theta_{1}}{\frac{\mu_{1}}{\mu_{2}} n_{2}^{2} \cos \theta_{1}+n_{1} \sqrt{n_{2}^{2}-n_{1}^{2} \sin ^{2} \theta_{1}}} .
\end{gathered}
$$

对于入射光电矢量垂直于入射平面的 $s$ 分量, 反射系 数 $r_{s}$ 和透射系数 $t_{s}$ 为

$$
\begin{aligned}
& r_{s}=\frac{\vec{E}_{s}^{\prime \prime}}{\vec{E}_{s}}=\frac{n_{1} \cos \theta_{1}-\frac{\mu_{1}}{\mu_{2}} \sqrt{n_{2}^{2}-n_{1}^{2} \sin ^{2} \theta_{1}}}{n_{1} \cos \theta_{1}+\frac{\mu_{1}}{\mu_{2}} \sqrt{n_{2}^{2}-n_{1}^{2} \sin ^{2} \theta_{1}}}, \\
& t_{s}=\frac{\vec{E}_{s}^{\prime}}{\vec{E}_{s}}=\frac{2 n_{1} \cos \theta_{1}}{n_{1} \cos \theta_{1}+\frac{\mu_{1}}{\mu_{2}} \sqrt{n_{2}^{2}-n_{1}^{2} \sin ^{2} \theta_{1}}} .
\end{aligned}
$$

如入射角 $\theta_{1}$ 不变的, 形成油膜的油种固定, 因此 

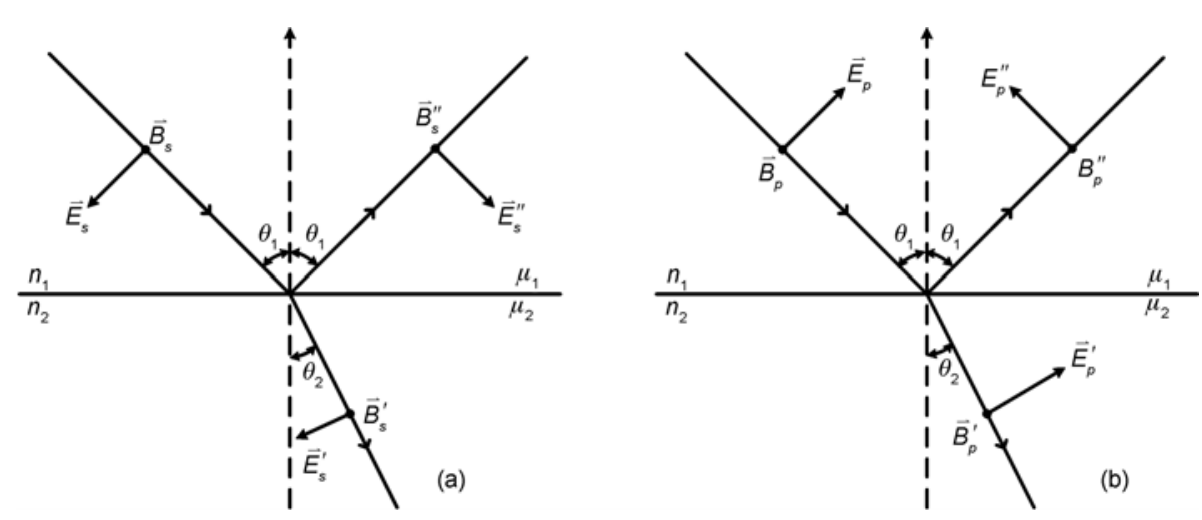

图 2 入射光在油膜界面的反射与折射

(a) 垂直于入射面的偏振; (b) 平行于入射面的偏振

介质折射率为 $n_{1}$ 与 $n_{2}$, 磁导率 $\mu_{1}$ 与 $\mu_{2}$ 都可视为常数 项. 由公式(8) (11) 可知, 油膜上下表面的反射系数 和透射系数取决于入射光电矢量的偏振方向、入射角 以及介质的物理特性参数, 可以将油膜两个表面上 的反射率和折射率看成为统计平均数. 因此公式(4) 和(5)中参量 $A_{0}, A_{1}, A_{3}, A_{4}$ 在每个波段是以一个常数 形式存在.

参量 $A_{2}$ 中含有油膜对入射光的消光系数 $\alpha$, 且与 油膜厚度 $d$ 密切相关. 入射光在油膜中的传播过程, 在局部可视为入射光与油膜中油分子团的相互作用 过程，该参量可以反映油膜中油分子团的对入射光 的分子散射性质, 因此油膜反射率也进一步反映出 油膜厚度变化的光谱响应信息. 入射光在油膜中传 播过程中分为两部分, 一部分为油分子团透射光; 另 一部分是被油分子团散射至 $4 \pi$ 立体角的散射光, 散 射光脱离了光线的传播方向, 这部分散射能量可以 认为损失了. 沿光线传播方向上, 由于散射而损失掉 的能量可以写成:

$$
\mathrm{d} I(s)=I(s+\mathrm{d} s)-I(s),
$$

其中 $\mathrm{d} s$ 为传播路径方向上的微分长度.

入射光的散射与介质直径大小具有密切的关系, 影响着探测目标的遥感响应 ${ }^{[29 ~ 31]}$. 散射强度一般是 入射光波长 -2 次方 $\left(\lambda^{-2}\right)$ 的函数 $a=f\left(\lambda^{-2}\right)$, 如米氏散 射、瑞利散射强度分别与 $\lambda^{-2} 、 \lambda^{-4}$ 成正比 ${ }^{[32]}$. 同时, 从 沿传播方向的微观角度来看, 散射光强度和入射光 强度 $I$ 成正比, 同传播路径长度 $\mathrm{d} s$ 成正比, 建立微分 方程来描述这种能量损失的过程, 如公式(13):

$$
I(s+\mathrm{d} s)-I(s)=d I(s)=-I(s) f\left(\lambda^{-2}\right) \mathrm{d} s .
$$

$$
I(s)=I_{0} \mathrm{e}^{-f\left(\lambda^{-2}\right) s},
$$

其中 $I_{0}$ 为入射光初始强度.

对于散射函数 $a=f\left(\lambda^{-2}\right)$, 可以利用泰勒公式将公 式(14)在 0 点展开为

$$
a=f\left(\lambda^{-2}\right)=a_{0}+a_{1} \lambda^{-2}+a_{2} \lambda^{-4} .
$$

其中 $a_{0} \sim a_{2}$ 为常数项, 公式(15)带入公式(6)可以得到

$$
A_{2}=\frac{a s}{d}=\frac{2 a}{\cos \theta}=\frac{2}{\cos \theta}\left(a_{0}+a_{1} \lambda^{-2}+a_{2} \lambda^{-4}\right) .
$$

公式(16)中 $(2 / \cos \theta)$ 项, 因为角度是固定的, 可以视为 常数项, 因此公式(16)可以写成:

$$
A_{2}=a_{0}^{\prime}+a_{1}^{\prime} \lambda^{-2}+a_{2}^{\prime} \lambda^{-4},
$$

其中 $a_{0}^{\prime} \sim a_{2}^{\prime}$ 为常数项.

以上分析表明, 在油膜双光束干涉模型中 $A_{0}, A_{1}$, $A_{3}$ 和 $A_{4}$ 在每个光谱段中都可以看为常数; $A_{2}$ 是该模 型中关键参量, 油膜厚度的变化导致了油膜中光散 射强度的变化, 而油膜中光散射强度又与油膜中消 光系数具有明显的关联性, 因此, 深入探讨油膜的光 学散射特性在油种遥感识别与油膜厚度遥感反演等 方面具有重要的意义.

\section{3 数据分析与验证}

\section{1 数据来源与预处理}

数据来源于海面油膜光谱响应实验, 该实验以辽 东湾海水与辽河油田原油为材料, 利用 ASD 全谱段地 物光谱仪, 针对海洋石油污染中的海面较薄油膜为研 究对象, 模拟海面油膜厚度连续变化过程中的油膜反 射光谱变化. 在海水表面连续滴入原油, 形成厚度不 
断变化的原油油膜, 在两台 $500 \mathrm{~W}$ 的溴铇灯光的照射 下, 利用 ASD 地物光谱仪进行光谱测量, ASD 地物光 谱仪测量范围为 350 2500 $\mathrm{nm}$, 光谱分辨率为 3 $\mathrm{nm}(350 \sim 1050 \mathrm{~nm})$ 和 $10 \mathrm{~nm}(1000 \sim 2500 \mathrm{~nm})$, 获取海面 油膜厚度连续变化中的油膜反射光谱, 对每次测量的 5 条曲线求平均, 对其作 9 点平滑处理. 前期的研究已 经表明了油膜光谱反射率与油膜的厚度呈很强的幂函 数负相关关系, 以 550 和 $645 \mathrm{~nm}$ 为中心的绿光、红光 波段的油膜光谱响应表现最优, 可作为海面油膜多/高 光谱遥感探测与评估的最佳选择波段 ${ }^{[12]}$. 本研究在前 期研究基础上, 选择有效谱段范围内(380 760 nm), 共计 49 条反射光谱数据(背景水体光谱 1 条, 油膜光谱 数据 48 条)作为研究基础数据, 如图 3 所示.

实验中以滴入的油滴数来代表油膜厚度, 油膜 厚度正比于滴入的油滴数 $N$, 在滴入设备的控制下, 每一滴的油滴量认为是相同的, 实验中容器面积与 探测面积是固定的, 可以认为油膜厚度 $d$ 正比于油滴 数 $N$, 即 $d \propto N$, 则 $d=m N$, 公式(2)可以写成:

$$
\left\{\begin{array}{l}
\Delta=2 n_{2} m N \cos \theta, \\
s=\frac{2 m N}{\cos \theta} .
\end{array}\right.
$$

参数 $A_{2}$ 和 $A_{3}$ 因此可以写为

$$
\begin{aligned}
& A_{2}=\frac{a s}{d}=\frac{a s}{m N}=\frac{2 a}{\cos \theta}, \\
& A_{3}=\frac{k \Delta}{d}=\frac{k \Delta}{m N}=2 k n_{2} \cos \theta .
\end{aligned}
$$

则模型公式(5)可以进一步写为以油滴数 $N$ 为自变量, 油膜反射率为因变量的公式:

$$
R=A_{0}+A_{1} \mathrm{e}^{-A_{2} N} \cos \left(A_{3} N\right)+A_{4} \mathrm{e}^{-2 A_{2} N} .
$$

在 IDL 软件平台中, 利用实验数据, 结合公式 (21), 以滴入油滴数与反射率值为变量, 计算 $A_{0} \sim A_{4}$ 在 381 760 nm 光谱范围内的值, 具体计算过程略.

\section{2 浮油膜的光散射特性分析}

浮油膜可以视为光学不均匀性较为显著的浑浊 介质, 油膜中的油分子团作为散射微粒, 其大小对入 射光的散射具有一定的影响. 根据散射理论, 如微粒 大小远小于波长, 其散射特点就符合瑞利散射, 散射 强度与入射光波长的四次方成反比, 即 $I \propto \lambda^{-4}$, 瑞 利散射在较短波长(即蓝-绿-红可见光波段)时具有意 义; 当微粒大小接近或者大于波长时, 其散射规律就 和瑞利散射不同, 符合米氏散射规律, 散射强度随波 长的变化规律是与入射光波长的较低幂次成反比, 即 $I \propto \lambda^{-2}$. 一般情况下, 认为米氏散射的散射强度 与入射光波长的二次方成反比, 但是如果因为介质 中浓度起伏, 引起介质光学性质的非均匀, 产生光的 分子散射, 分子散射中散射强度与波长的关系与瑞 利散射相同 ${ }^{[32]}$.

模型参量 $A_{2}$ 包含了油膜的散射特性, 分析 $A_{2}$ 与 波长 $\lambda$ 的关系, 可用来验证理论中该参量的特性, 即 油膜散射特性的假设. 以波长 $\lambda$ 为自变量 $x, A_{2}$ 为因变 量 $y$, 按照公式(17)进行曲线拟合分析, 如图 4 所示,

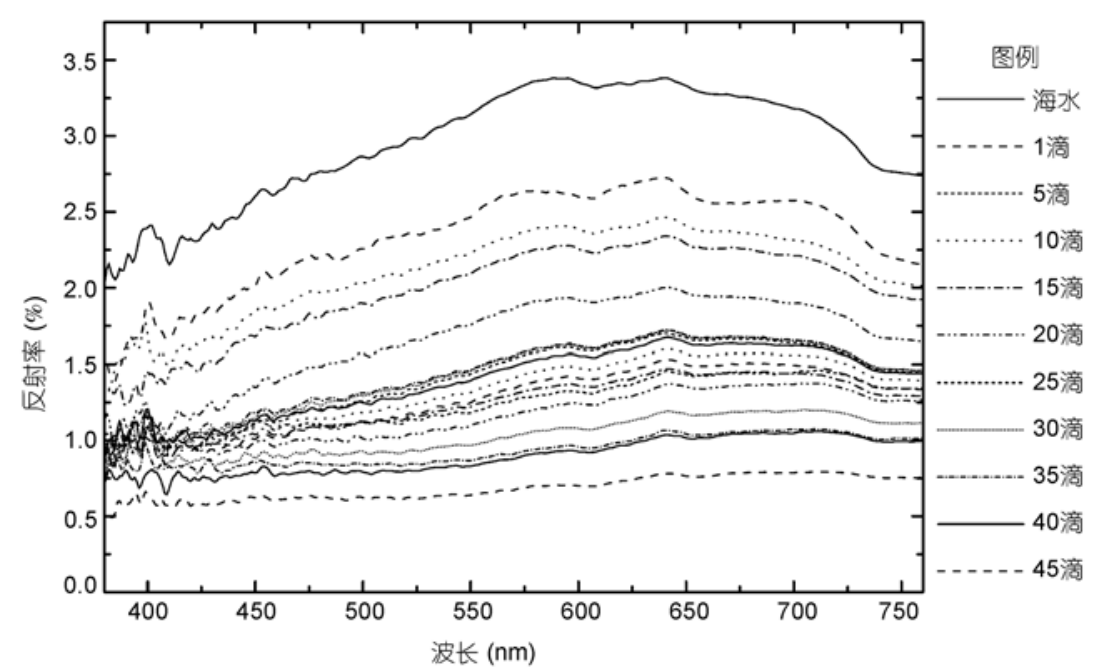

图 3 不同油膜厚度的光谱反射率 

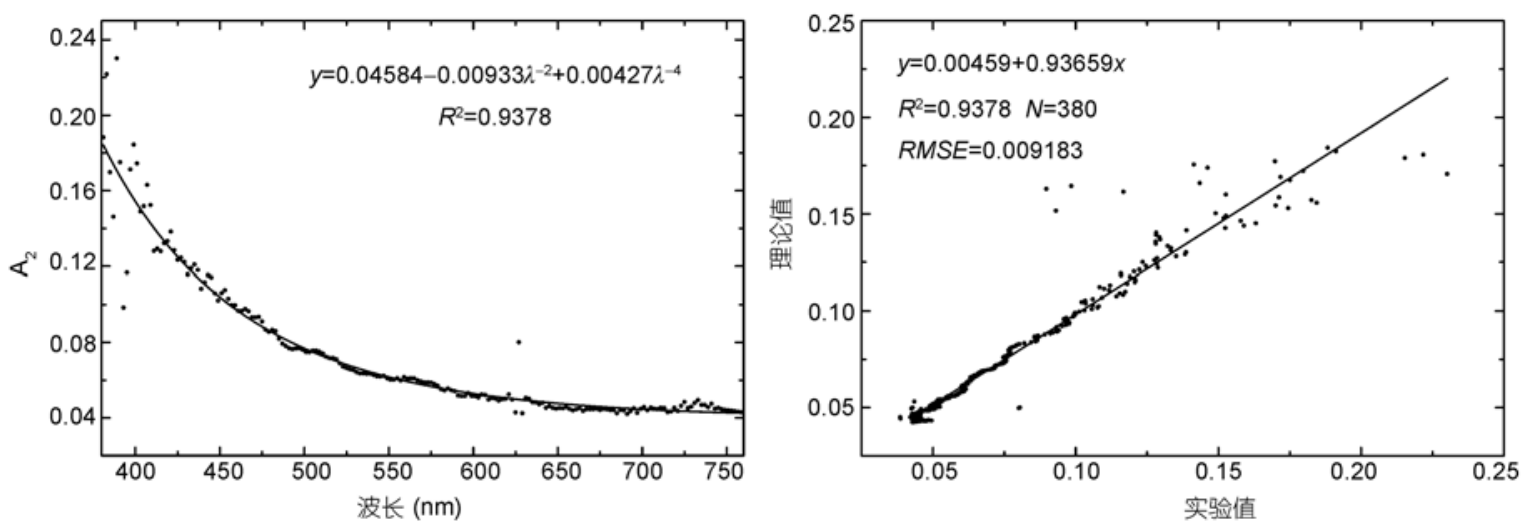

图 4 浮油膜的分子散射特性数据分析

参数 $A_{2}$ 与波长 $\lambda$ 的关系较好的符合了公式(17)的规律, 其拟合表达式为

$$
y=0.04584-0.00933 \lambda^{-2}+0.00427 \lambda^{-4} .
$$

$R^{2}=0.9378$, 统计量 $N=380(381 \sim 760 \mathrm{~nm}$ 波段、分辨率 为 $1 \mathrm{~nm}$, 共 380 个数据), $R M S E=0.009183$. 以上分析 表明: 油膜的双光束干涉模型参量 $A_{2}$ 与波长具有密 切的关联性, 原油油膜中油分子团的大小不等, 油膜 的散射较为复杂, 油膜对入射光的散射作用是其反 射率发生变化的根本原因, 成为海面油膜厚度遥感 反演的理论依据.

\section{3 基于模型的油膜反射率随厚度变化响应}

油膜实验中, 油膜光谱反射率明显低于海水本 底反射率, 并随着油膜厚度的增加而逐步降低, 考虑 水体和海面油膜在可见光波段对太阳光的反射能力 强而在近红外波段反射能力较弱, 以及大气和海水 对蓝色波段反射率的影响, 对海面油膜光谱响应较 强的绿光波段 (中心波长为 $550 \mathrm{~nm}$ ) 和红光波段(中心 波长为 $645 \mathrm{~nm}$ )作为海面较薄油膜探测与评估的多/ 高光谱遥感最佳选择波段 ${ }^{[12]}$, 以滴入油滴数 $N$ 为自 变量, 模型公式(21)为方程式, $A_{0}, A_{1}, A_{3}, A_{4}$ 为统计得 到的常数项, 参数 $A_{2}$ 按照公式(22)计算得到, 模拟随 油膜厚度(滴入油滴数代替)变化情况下, 油膜反射光 谱值, 以 550 和 $645 \mathrm{~nm}$ 波段为例, 验证并评价本研究 的模型模拟值精度.

如图 5 所示, 550 和 $645 \mathrm{~nm}$ 处模型的预测反射率 值与实测油膜反射值取得较高的一致性, 其中 550 $\mathrm{nm}$ 处的复相关系数 $R^{2}=0.9091, R M S E=0.774 ; 645 \mathrm{~nm}$
处的复相关系数 $R^{2}=0.8952, R M S E=0.871$, 对比前期 研究结论 ${ }^{[14]}$, 表明该模型保持了较高的拟合精度, 也 表明浮油膜双光束干涉模型有效地解释了海面浮油 膜光谱反射率对其厚度变化响应的原理.

\section{4 结论}

(1) 将均匀分布的浮油膜视为一个平行平板, 分 析油膜对入射光的辐射传输过程, 建立基于油膜双 光束干涉的油膜厚度遥感定量反演模型, 同时结合 油膜光谱响应实验数据进行模型的验证与分析, 表 明油膜厚度遥感定量反演模型不仅具有明确的理论 意义，也具有良好的模拟精度.

(2) 结合菲涅尔公式, 分析浮油膜双光束干涉模 型中参量的物理意义, 表明对单一油种构成的油膜 而言, 如入射光角度不变, 该模型参数在每个谱段上 都可以视作一个固定值, 因此在本模型基础上, 建立 典型油种油膜的模型参数查找表, 将有效提高该模 型的应用效能.

(3) 油膜的消光特性是今后海面浮油膜遥感研 究需要关注的核心问题之一, 本研究中该参数的理 论意义与数据分析表明, 浮油膜对入射光具有分子 散射作用, 因此油膜厚度变化产生了反射光谱响应. 考虑海面浮油膜来源多样, 其构成油种的光学散射 特性不同, 所以该参数的深入研究将有助于海面浮 油膜的识别和判定. 此外, 海水中悬浮物浓度、叶绿 素含量和黄色物质等不同, 会产生复杂的海水背景 影响, 因此今后的研究与应用中还需要考虑背景影 响因素的剔除. 

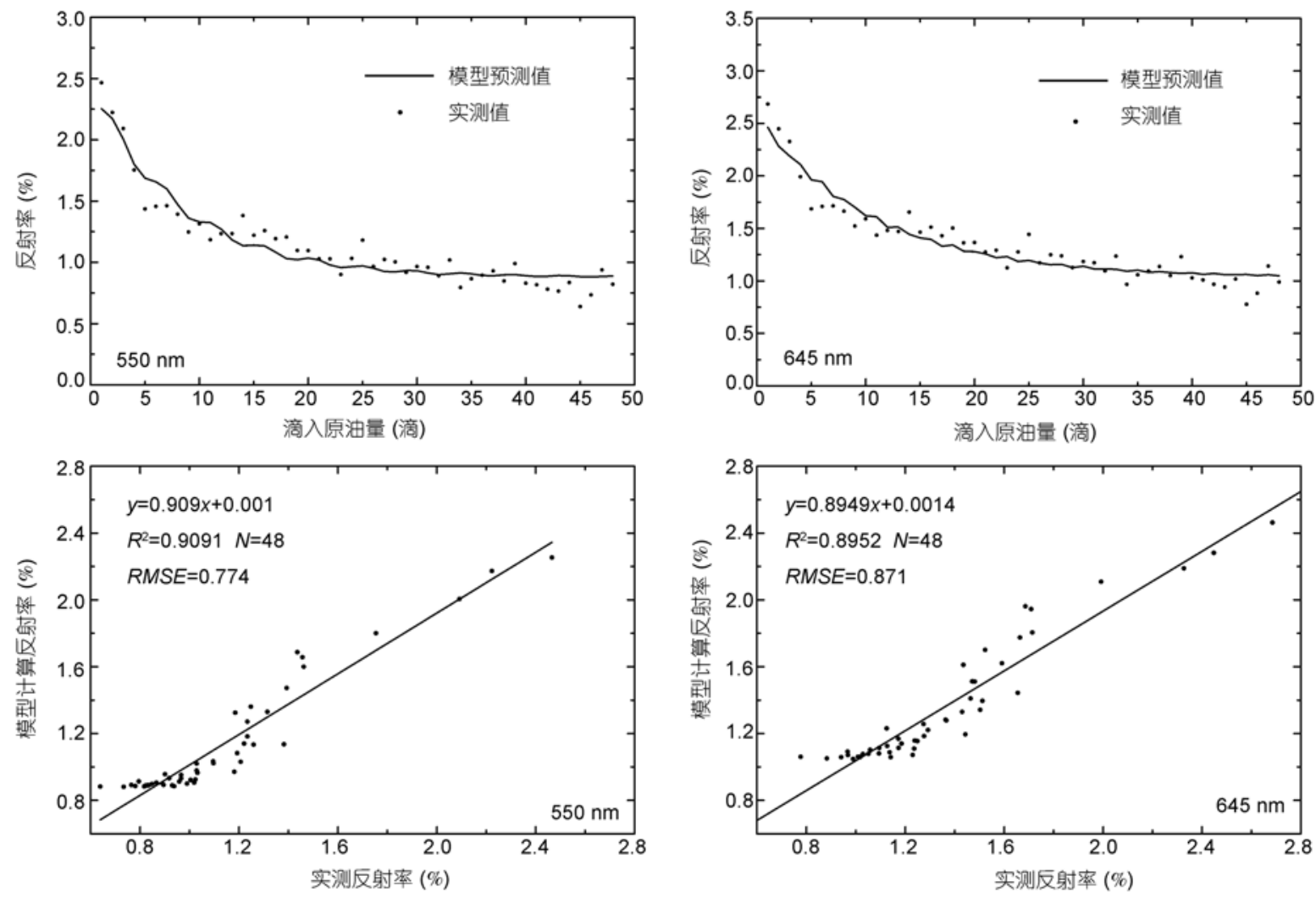

图 5 浮油膜双光束干涉模型的模拟与验证 550 和 $645 \mathrm{~nm}$ 反射率的模型预测值与实测值

\section{参考文献}

1 Sackett W M. Use of hydrocarbon sniffing in offshore exploration. J Geochem Explor, 1977, 7: 243-254

2 Acinas J R, Brebbia C A, eds. Computer Modeling of Seas and Coastal Regions III. Southampton Boston: Computation Mechanics Publication, 1997

3 O'Briena G W, Lawrenceb G M, Williamsc A K, et al. Yampi Shelf, Browse Basin, North-West Shelf, Australia: A test-bed for constraining hydrocarbon migration and seepage rates using combinations of 2D and 3D seismic data and multiple, independent remote sensing technologies. Mar Pet Geol, 2005, 22: 517-549

4 Kvenvolden K A, Cooper C K. Natural seepage of crude oil into the marine environment. Geo Mar Lett, 2003, 23: 140-146

5 Palmer D, Boasted G A, Boxall S R. Airborne multi spectral remote sensing of the January 1993 Shetlands oil spill. In: Proceedings of the Second Thematic Conference on Remote Sensing for Marine and Coastal Environments: Needs, Solutions and Applications, ERIM Conferences, Ann Arbor, 1994. II -546-558

6 Foudan M F S. Hyperspectral remote sensing: A new approach for oil spill detection and analysis. Doctoral Dissertation. Fairfax: George Mason University, 2003

7 赵冬至，从丕福. 海面溢油的可见光波段地物光谱特征研究. 遥感技术与应用, 2000, 15: 160-164

8 张永宁, 丁倩, 李栖筠. 海上溢油污染遥感监测的研究. 大连海事大学学报, 1999, 25: 1-5

9 张永宁, 丁倩, 高超, 等. 油膜波谱特征分析与遥感监测溢油. 海洋环境科学, 2000, 19: 5-10

10 陆应诚, 田庆久, 宋鹏飞, 等. 海面油膜高光谱遥感信息提取. 遥感学报, 2009, 13: 691-695 
11 陆应诚, 田庆久, 齐小平, 等. 海面甚薄油膜光谱响应研究与分析. 光谱学与光谱分析, 2009, 29: 986-989

12 陆应诚, 田庆久, 王晶晶, 等. 海面油膜光谱响应实验研究. 科学通报, 2008, 53: 1085-1088

13 付玉慧, 李栖篛, 张宝茹. 海洋溢油光谱分析与卫星信息提取. 遥感学报, 2008, 12: 1010-1016

14 Magnus W, Paul J D, Graham A L, et al. Assessing the effect of hydrocarbon oil type and thickness on a remote sensing signal: A sensitivity study based on the optical properties of two different oil types and the Hymap and Quickbird sensors. Remote Sens Environ, 2009, 113: 2000-2010

15 Fingas M F, Brown C E. Review of oil spill remote sensing. In: Proceedings of the Fifth International Conference on Remote Sensing for Marine and Coastal Environment. Ann Arbor: Environmental Research Institute of Michigan, 2000. I-211-218

16 Fingas M F, Brown C E, Mullin J V. The visibility limits of oil on water and remote sensing thickness detection limits. In: Proceedings of the Fifth Thematic Conference on Remote Sensing for Marine and Coastal Environments. Ann Arbor: Environmental Research Institute of Michigan, 1998. II-411-418

17 黄晓霞, 朱振海. 海洋表面膜特征的 SAR 图像探测. 遥感学报, 1999, 3: 48-53

18 Mussetto M S, Yujiri L, Dixon D P, et al. Passive millimeter wave radiometric sensing of oil spills. In: Proceedings of the Second Thematic Conference on Remote Sensing for Marine and Coastal Environments: Needs, Solutions and Applications, ERIM Conferences, Ann Arbor, Michigan, 1994. 35-46

19 Bern T I, Wahl T, Anderssen T, et al. Oil spill detection using satellite based SAR: Experience from a field experiment. Photogramm Eng Remote Sens, 1993, 59: 423-428

20 Balick L, DiBenedetto J A, Lutz S S. Fluorescence emission spectral measurements for the detection of oil on shore. In: Proceedings of the Fourth Thematic Conference on Remote Sensing for Marine and Coastal Environments. Ann Arbor: Environmental Research Institute of Michigan, 1997. 13-20

21 Pantani L, Cecchi G, Bazzani M. Remote sensing of marine environments with the high spectral resolution fluorosensor, FLIDAR 3. SPIE, 1995, 2586: 56-64

22 Campbell I, McStay D. A ship-borne system for the detection of surface oil. In: Advanced Technologies for Environmental Monitoring and Remediation. Bellingham, Washington: The International Society for Optical Engineering, 1995. 214-219

23 Piskozub J, Drozdowska V, Varlamov V. A lidar system for remote measurement of oil film thickness on sea surface. In: Proceedings of the Fourth Thematic Conference on Remote Sensing for Marine and Coastal Environments. Ann Arbor: Environmental Research Institute of Michigan, 1997. 386-391

24 O'Neil R A, Neville R A, Thompson V. The arctic marine oil spill program (AMOP) remote sensing study. Environment Canada Report EPS 4-EC-83-3, Ottawa, Ontario, 1983

25 Brown H M, Bittner J P, Goodman R H. The limits of visibility of spilled oil sheens. In: Proceedings of the Second Thematic International Airborne Remote Sensing Conference and Exhibition. Ann Arbor: Environmental Research Institute of Michigan, 1996. III-327-334

26 Taylor S. 0.45 to $1.1 \mu \mathrm{m}$ spectra of prude crude oil and of beach materials in Prince William Sound. Alaska, CRREL Special Report No. 92-5. Hanover: Cold Regions Research and Engineering Laboratory, 1992. 14

27 马科斯 波恩, 埃米尔 沃尔夫, 著. 杨臀荪, 等, 译. 光学原理. 第七版. 北京: 电子工业出版社, 2005. 32-299

28 赵建林, 编. 高等光学. 北京: 国防工业出版社, 2002. 35-40

29 梁顺林, 著. 范闻捷, 译. 定量遥感. 北京: 科学出版社, 2009. 24-32

30 赵英时, 编著. 遥感应用分析原理与方法. 北京: 科学出版社, 2003. 20-22

31 徐希孺, 著. 遥感物理. 北京: 北京大学出版社, 2005. 198-199

32 石顺祥, 张海兴, 刘劲松, 著. 物理光学与应用光学. 西安: 西安电子科技大学出版社, 2000. 276-290 